\title{
Paneles unidos de canto y contrachapados fabricados con madera juvenil de Eucalyptus nitens*
}

Edge-glued panels and plywoods manufactured with juvenile Eucalyptus nitens wood

\author{
JUSTO LISPERGUER $\mathrm{M}^{1}$., CARLOS ROZAS M. ${ }^{2}$ \\ ${ }^{1}$ Departamento de Química, Facultad de Ciencias, Universidad del Bío Bío. \\ E-mail: jlisperg@ubiobio.cl \\ 2 Departamento Ingeniería en Maderas, Facultad de Ingeniería. Universidad del Bío Bío. \\ Casilla 5C. Concepción, Chile.
}

\begin{abstract}
SUMMARY
This work evaluates the bonding quality in solid wood of juvenile (11 years) E. nitens. Edge-glued panels were manufactured and bonded with PVA (Polyvinyl acetate). The mechanical properties of the panels were determined and compared with those of mature (25 years) $P$. radiata panels. Shear strength values in $E$. nitens panels were found to be 35\% higher than $P$. radiata panels according to British Standard EN 204-205 on non-structural adhesives for wood joining. Juvenile $E$. nitens wood venners were used to manufacture exterior -grade plywood panels. Laboratory results demonstrated that E. nitens plywood satisfies all the British Standard Institution requirements for exterior use.
\end{abstract}

Key words: edge glued-panels, plywoods, E. nitens, bond quality.

\section{RESUMEN}

El presente trabajo evalúa la calidad de la unión adhesiva en madera sólida de Eucalyptus nitens de 11 años. Con esta finalidad se fabricaron paneles unidos de canto utilizando PVA (acetato de polivinilo) como adhesivo. Se midieron las propiedades físico-mecánicas de estos paneles y se compararon con las propiedades de paneles unidos de canto fabricados con madera de Pinus radiata de 25 años. Como resultado de estos ensayos se encontró que los paneles a base de E. nitens presentan valores promedio de resistencia al cizalle superiores en un $35 \%$ a los paneles de $P$. radiata de acuerdo a condiciones de uso en interiores de la norma British Standard EN 204205 para adhesivos no estructurales. Se fabricaron también chapas de madera juvenil de E. nitens para elaborar tableros contrachapados de uso exterior. Los resultados de laboratorio demuestran que estos tableros satisfacen todos los requerimientos de las normas British Standard Institution para uso exterior.

Palabras clave: paneles unidos de canto, contrachapados, E. nitens, calidad adhesiva.

\footnotetext{
* Financiado por Fund for the Promotion of Scientific and Technological Development (FONDEF 98 I 1018).
} 


\section{INTRODUCCION}

Eucalyptus nitens es una de las especies introducidas en Chile en la década de los años 60, junto a otras especies de la misma familia y de algunas coníferas. Esta especie ha ganado interés por su rápido crecimiento en la zona central de Chile (11-15 años), donde alcanza densidades similares a la madera de Pinus radiata maduro y un importante rendimiento volumétrico entre 20 y 40 $\mathrm{m}^{3} /$ ha por año (1). El uso de esta especie se ha destinado preferentemente a procesos de pulpaje para obtención de celulosa a pesar de que rollizos destinados a madera aserrable y debobinable tienen valores que casi duplican el valor del rollizo pulpable (2). Estudios de las características físico-mecánicas de E. nitens de 12 años lo clasifican como una madera liviana de densidad alrededor de $500 \mathrm{~kg} / \mathrm{m}^{3}$, semidura y resistencia a la flexión estática de alrededor de $1.200 \mathrm{~kg} / \mathrm{cm}^{2}$ (3).

El uso de E. nitens en la industria de productos manufacturados reconstituidos en Chile es prácticamente nulo. Uno de los problemas es la pérdida por colapso durante el secado y escaso conocimiento del comportamiento de la unión adhesiva, frente a adhesivos tradicionales como acetato de polivinilo (PVA), urea formaldehído (UF) y fenol formaldehído (PF). El uso en tableros contrachapados y vigas laminadas de especies de Eucalyptus de rápido crecimiento ha sido estudiado con interés en países como Australia y Nueva Zelandia, donde se informa que Eucalyptus regnans y Eucalyptus grandis han sido empleados con éxito en varios tipos de elementos manufacturados reconstituidos $(4,5)$. Estudios realizados en Chile por Guzmán et al. (6) sobre las características de encolabilidad de especies como Eucalyptus globulus, Eucalyptus nitens y Eucalyptus regnans muestran que este tipo de madera desarrolla un buen comportamiento de resistencia al cizalle de la unión adhesiva en uso para interiores y que la madera adulta supera en todos los casos el comportamiento de madera juvenil.

Tableros con madera sólida unida de canto y contrachapados son extensamente usados en Chile y constituyen un importante volumen de las exportaciones. Estos elementos son fabricados con madera adulta de $P$. radiata. Las densidades básicas similares entre $P$. radiata adulto y $E$. nitens de 11 años de alrededor de $450-500 \mathrm{~kg} / \mathrm{m}^{3}$ y las características estéticas de la madera de este últi- mo fundamentan el presente estudio orientado a evaluar la calidad de la unión adhesiva de tableros unidos de canto y contrachapados fabricados con madera juvenil de $E$. nitens y compararla con el comportamiento de estos mismos elementos fabricados con madera adulta de $P$. radiata.

\section{MATERIAL Y METODOS}

La madera de Eucalyptus nitens fue obtenida de árboles de 11 años de edad con DAP $47 \mathrm{~cm}$ provenientes del fundo Nihuinco, Mulchén, VIII Región (Chile). De cada árbol se obtuvieron trozas de $3 \mathrm{~m}$ de longitud medidos a partir de los $0,3 \mathrm{~m}$ de altura y de cada troza se extrajeron rodelas para la determinación de la densidad básica por la norma NCh 176/2 (7). La madera fue aserrada y secada por un método convencional que consideró una temperatura inicial de $30^{\circ} \mathrm{C}$, con procesos de calentamiento por 4 horas, un reacondicionado para recuperación del colapso, un igualado y un acondicionado de la madera para igualar el contenido de humedad y liberar las tensiones de secado. El tiempo de secado fluctuó entre 29-45 días desde un contenido de humedad inicial de $140 \%$ hasta un contenido de humedad final de $10 \%$.

Paneles unidos de canto: Se fabricaron paneles formados por diez piezas de madera sólida al $12 \%$ de humedad y libre de defectos, con dimensiones de 22 × 45 × $1.700 \mathrm{~mm}$ en el espesor, ancho y largo respectivamente. Estudios de microscopia de superficies de madera cepillada y aserrada revelan diferente penetrabilidad del adhesivo en estas superficies, que puede afectar la calidad de la unión (8). Para estudiar el efecto de este parámetro se elaboraron cuatro paneles con piezas aserradas y cuatro paneles con piezas cepilladas en el canto para analizar la influencia de la calidad superficial en la unión adhesiva.

Para estudiar la posible influencia del tipo de corte se fabricaron también cuatro paneles de madera aserrada provenientes de corte radial y cuatro paneles con madera de corte tangencial, lo que hizo un total de 16 paneles a base de E. nitens.

Para comparar propiedades en las condiciones de laboratorio se fabricaron también cuatro paneles con madera de $P$. radiata de 25 años. Los paneles fueron encolados con un adhesivo PVA comercial con un esparcido de $180 \mathrm{~g} / \mathrm{m}^{2}$ en una 
sola línea de cola y prensados durante tres horas a $7 \mathrm{~kg} / \mathrm{cm}^{2}$ a temperatura ambiente de $20^{\circ} \mathrm{C}$ en el Centro de Alta Tecnología de la Madera (CATEM) de la Universidad del Bío Bío. Para los ensayos de resistencia al cizalle se utilizó la norma British Standard EN 204-205 $(9,10)$, que es exigida por los mercados asiáticos para estos productos fabricados a base de $P$. radiata.

Tableros contrachapados: Para la fabricación de chapas se utilizaron trozas de $E$. nitens de 11 años, de $120 \mathrm{~cm}$ de largo x $40 \mathrm{~cm}$ de diámetro, las cuales fueron maceradas en agua utilizando un programa que permitió alcanzar una temperatura de $75{ }^{\circ} \mathrm{C}$ en el rollete residual al cabo de 55 horas de macerado, posteriormente el trozo fue debobinado, dimensionado a chapas de $2 \times 550$ x $1.000 \mathrm{~mm}$ y secadas a un contenido de humedad de $8 \%$. Se fabricaron tableros de tres capas de 500 x $500 \mathrm{~mm}$ utilizando como adhesivo una resina comercial fenol-formaldehído de comportamiento exterior. Las condiciones de prensado fueron: presión máxima $12 \mathrm{~kg} / \mathrm{cm}^{2}$, una temperatura de platos de $140{ }^{\circ} \mathrm{C}$ y un tiempo de prensado de 1 $\mathrm{min} / \mathrm{mm}$. Se midieron las propiedades físico-mecánicas de los tableros, evaluando su resistencia al cizalle y a la flexión estática mediante las normas British Standard 6566(11) y DIN 52371 (12) en una máquina de ensayos universal Instron de $5.000 \mathrm{~kg}$.

\section{RESULTADOS Y DISCUSION}

La densidad básica promedio de Eucalyptus nitens varía desde 496 a $598 \mathrm{~kg} / \mathrm{m}^{3}$ para alturas entre 0,3 y 30,3 m. El Pinus radiata de 25 años presenta una densidad básica promedio de 475 $\mathrm{kg} / \mathrm{m}^{3}$. En el cuadro 1 se muestran los valores promedio de esfuerzos de cizalle de paneles unidos de canto a base de Eucalyptus nitens de diferente tipo de corte (radial y tangencial) y diferente calidad superficial en la línea de unión (madera aserrada y cepillada) y paneles a base de madera adulta de Pinus radiata, todos encolados con PVA.

La norma BS EN 204 define las clases de durabilidad para el comportamiento de uniones adhesivas: $\mathrm{D}_{1}$, interior expuesto a cortos períodos de humedad; $\mathrm{D}_{2}$, interior con períodos cortos y ocasionales de exposición al agua condensada y alta humeda; $\mathrm{D}_{3}$, interior con frecuentes exposiciones al agua condensada o ambientes exteriores cubiertos. Los mercados externos, especialmente el mercado asiático, exigen la durabilidad $\mathrm{D}_{3}$ para este tipo de tableros. Con excepción de la clase $\mathrm{D}_{1}$, los resultados mostrados en el cuadro 1 para las condiciones $\mathrm{D}_{2}$ y $\mathrm{D}_{3}$ superan los requerimientos de la norma que son de 8 y $2 \mathrm{~N} / \mathrm{mm}^{2}$ respectivamente. La durabilidad $\mathrm{D}_{1}$ requiere una resistencia al esfuerzo de cizalle de $10 \mathrm{~N} / \mathrm{mm}^{2}$ que no fue alcanzada por ninguno de los dos tipos de tableros debido a que se produjo falla de madera antes de alcanzar este valor. Esta situación ha sido informada por fabricantes de adhesivos PVA que se utilizan habitualmente en el sector de remanufacturas a base de madera de Pinus radiata (13).

Los paneles unidos de canto, fabricados con madera de $E$. nitens, muestran valores promedio de resistencia al cizalle $35 \%$ superiores a los paneles de $P$. radiata para las condiciones de durabilidad $\mathrm{D}_{1}$ y $\mathrm{D}_{2}$. Para las condiciones de durabilidad $\mathrm{D}_{3}$ no existen diferencias significativas entre paneles fabricados con una u otra especie, dado que la resistencia mínima exigida para esta condición es sólo de $2 \mathrm{~N} / \mathrm{mm}^{2}$. Tampoco existen diferencias significativas entre paneles fabricados con madera de E. nitens provenientes de corte radial y corte tangencial. En igual forma, el análisis estadístico revela que no existen diferencias significativas entre paneles de $E$. nitens con superficies de unión aserradas y cepilladas. El cuadro 2 presenta los resultados de resistencia al cizalle, módulo de elasticidad (MOE) y módulo de ruptura (MOR) de tableros contrachapados fabricados con madera de E. nitens de 11 años.

El valor promedio de resistencia al cizalle en tableros contrachapados fue de $1,43 \mathrm{~N} / \mathrm{mm}^{2}$, para el ensayo sin envejecimiento y de $1,02 \mathrm{~N} / \mathrm{mm}^{2}$ para el ensayo después de sumergir las probetas a 72 horas en agua a $100^{\circ} \mathrm{C}$. Estos valores se encuentran dentro del rango aceptado para adhesivos de uso exterior por la norma British Standard 6566. El valor mínimo aceptable para este tipo de tableros es de $0,35 \mathrm{~N} / \mathrm{mm}^{2}$. Los valores promedio del MOR de 113,90 también se encuentran sobre los mínimos exigidos por la norma de 20-40 N/mm². El MOE de los tableros fue de $10.876 \mathrm{~N} / \mathrm{mm}^{2}$, que está en un rango aceptable sobre el mínimo. 


\section{CUADRO 1}

Valores de resistencia al cizalle de paneles unidos de canto de $E$. nitens y $P$. radiata encolados con PVA.

Values of shear strength of edge glued panels from $E$. nitens and $P$. radiata wood bonded with PVA.

\begin{tabular}{|c|c|c|c|c|c|c|}
\hline \multirow[b]{3}{*}{$\mathrm{n}$} & \multicolumn{3}{|c|}{ CEPILLADA } & \multicolumn{3}{|c|}{ ASERRADA } \\
\hline & $\mathrm{D}_{1}$ & $\mathrm{D}_{2}$ & $\overline{D_{3}}$ & $\overline{D_{1}}$ & $\mathrm{D}_{2}$ & $\overline{\mathrm{D}_{3}}$ \\
\hline & 20 & 20 & 20 & 20 & 20 & 20 \\
\hline \multicolumn{7}{|c|}{ E. nitens: tangencial } \\
\hline Cizalle N/mm² & 8,26 & 8,46 & 2,01 & 8,24 & 7,94 & 2,18 \\
\hline$\%$ Falla madera & 92 & 85 & 88 & 90 & 94 & 90 \\
\hline Desv. $\mathrm{St}^{1}$. & $(0,08)$ & $(0,20)$ & $(0,13)$ & $(0,30)$ & $(0,28)$ & $(0,12)$ \\
\hline \multicolumn{7}{|l|}{ E. nitens: radial } \\
\hline Cizalle N/mm² & 8,80 & 8,60 & 2,08 & 8,43 & 7,88 & 2,20 \\
\hline$\%$ Falla madera & 90 & 96 & 85 & 95 & 90 & 80 \\
\hline Desv. St. & $(0,14)$ & $(0,49)$ & $(0,02)$ & $(0,21)$ & $(0,31)$ & $(0,10)$ \\
\hline \multicolumn{7}{|l|}{ P. radiata } \\
\hline Cizalle N/mm² & 6,29 & 6,76 & 2,01 & & & \\
\hline$\%$ Falla madera & 96 & 94 & 82 & & & \\
\hline Desv. St. & $(0,38)$ & $(0,20)$ & $(0,15)$ & & & \\
\hline $\begin{array}{l}\text { Mínimo de norm } \\
\left(\mathrm{N} / \mathrm{mm}^{2}\right)\end{array}$ & $\geq 10,00$ & $\geq 8,00$ & $\geq 2,00$ & & & \\
\hline
\end{tabular}

n Número de probetas para cada acondicionamiento.

1 Desviación estándar.

$\mathrm{D}_{1} 7$ días en atmósfera estándar a $20^{\circ} \mathrm{C}$.

$\mathrm{D}_{2} 7$ días en atmósfera estándar +4 días en agua fría +7 días en atmósfera estándar.

$\mathrm{D}_{3} 7$ días en atmósfera estándar +4 días en agua fría.

\section{CUADRO 2}

Valores del módulo elasticidad (MOE) y módulo de ruptura (MOR) de tableros contrachapados de E. nitens en flexión estática.

Values of modulus of elasticity (MOE) and modulus of rupture (MOR) of E. nitens plywood in static bending.

\begin{tabular}{|lcccc|}
\hline Propiedades & $\mathrm{N}^{\circ}$ paneles & $\mathrm{N}^{\circ}$ probetas & Seco & 72 h ebullición \\
\hline Resistencia cizalle $\left(\mathrm{N} / \mathrm{mm}^{2}\right)$ & 5 & 100 & $1,43(0,156)$ & $1,02(0,389)$ \\
$\%$ Falla de madera & - & - & 87 & 80 \\
MOR $\left(\mathrm{N} / \mathrm{mm}^{2}\right)$ & 5 & 100 & - & $113,9(14,98)$ \\
MOE $\left(\mathrm{N} / \mathrm{mm}^{2}\right)$ & 5 & 100 & $10.879(1642)$ & - \\
\hline
\end{tabular}

En paréntesis la desviación estándar. 


\section{CONCLUSIONES}

El análisis de varianza de los resultados de este estudio demuestra que existen con un $95 \%$ de confianza diferencias significativas entre paneles unidos de canto fabricados con madera sólida de E. nitens de 11 años y paneles fabricados con madera de $P$. radiata de 25 años. Los paneles a base de Eucalyptus presentan en promedio propiedades mecánicas de resistencia al cizalle superiores en un $35 \%$ con relación a paneles unidos de canto fabricados a base de madera adulta de $P$. radiata, para condiciones de uso interior del tipo $\mathrm{D}_{1}$ y $\mathrm{D}_{2}$. En estos ensayos la madera de $P$. radiata falla antes de alcanzar los requerimientos mínimos de la norma EN 204. Para condiciones de uso exterior protegido tipo $\mathrm{D}_{3}$, el mismo análisis no muestra diferencias significativas entre ambos tipos de paneles debido a que la medición de resistencia al cizalle realizada con posterioridad al envejecimiento acelerado tiene un requerimiento mínimo de la norma muy inferior al de los tipos anteriormente señalados.

El estudio muestra además que no hay diferencias significativas entre paneles de E. nitens fabricados con madera cepillada o aserrada en el canto o cara de la unión adhesiva. Tampoco influye en la calidad adhesiva el tipo de corte radial o tangencial realizado para obtener la madera. Los tableros contrachapados de tres capas fabricados con chapas de E. nitens de 11 años y encolados con una resina fenol-formaldehído de uso exterior satisfacen todos los requerimientos de la norma British Standard 6566.

En conclusión, es posible fabricar paneles unidos de canto y tableros contrachapados de uso exterior a base de madera de E. nitens de 11 años con buenas propiedades para un uso final en la industria del mueble o la construcción.

\section{BIBLIOGRAFIA}

(1) NAVARRETE, P. Estudio para la utilización industrial de Eucalyptus nitens. Tesis de Ingeniería en Maderas. Universidad del Bío Bío. Concepción. Chile, 2002. p. 4.

(2) Instituto Forestal. Boletín de Precios Forestales. Chile, 2002, No 89. p. 5.

(3) PEREZ,V. Propiedades físico-mecánicas de E. fastigata, E. nitens y E. muricasta crecidos en Chile. Informe Técnico $N^{o}$ 97. Instituto Forestal. Chile, 1983, p. 46-52.

(4) HILLIS, W. Eucalyptus for wood production. Academic Press. Londres, 1984, pp 317-321.

(5) HASLETT, A., G. YOUNG. Nitens for sawn timber? New Zealand Tree Grower, 1992, vol. 12 N N $^{\circ}$, p. 8-9.

(6) GUZMAN, M., C. MOYA, G. HERNANDEZ, M. PEREDO. Alternativas industriales para el Eucalyptus globulus: Encolabilidad. Maderas: Ciencia y Tecnología, 2000, vol. 2, $\mathrm{N}^{\circ} 1$, p. 59-66.

(7) INSTITUTO NACIONAL DE NORMALIZACION, INN. Norma Chilena Oficial, 1988, NCh 176/2, p. 1-11.

(8) SINGH, A., C. ANDERSON, J. WARNES, J. MATSUMURA. The effect of planing on the microscopic structure of Pinus radiata wood cells in relation to penetration of PVA glue. Holz Roh-Werkstoff, 2002, vol. $60, \mathrm{~N}^{\circ} 5$, p. 333-341.

(9) BRITISH STANDARD INSTITUTION EN 204. Nonstructural adhesives for joining of wood and derived timber products. Londres. 1991. p. 1-8.

(10) BRITISH STANDARD INSTITUTION EN 205. Test methods for wood adhesives for non-structural applications-Determination of tensile shear strength of lap joints. Londres. 1991. p. 1-6.

(11) BRITISH STANDARD INSTITUTION. Plywood BS 6566: Part 8. Specification for bond performance of veneer plywood. Londres. 1985. p. 41-45.

(12) DEUTSCHES INSTITUT FÜR NORMUNG. DIN 52371. Testing of plywood, bending test. Berlin. 1968. p. 86-88.

(13) Anónimo. Informe Técnico. Adhesivos para remanufactura. Lignum (Chile), 2003, $\mathrm{N}^{\circ}$ 64, 21-24. 